\title{
Deaths by suicide and their relationship with general and psychiatric hospital discharge: 30-year record linkage study
}

Nadine Dougall, Paul Lambert, Margaret Maxwell, Alison Dawson, Richard Sinnott, Susan McCafferty, Carole Morris, David Clark and Anthea Springbett

\section{Background}

Studies have rarely explored suicides completed following discharge from both general and psychiatric hospital settings. such research might identify additional opportunities for intervention.

\begin{abstract}
Aims
To identify and summarise Scottish psychiatric and general hospital records for individuals who have died by suicide.
\end{abstract}

\section{Method}

A linked data study of deaths by suicide, aged $\geqslant 15$ years from 1981 to 2010 .

\section{Results}

This study reports on a UK data-set of individuals who died by suicide $(n=16411)$, of whom $66 \%(n=10907)$ had linkable previous hospital records. Those who died by suicide were 3.1 times more frequently last discharged from general than from psychiatric hospitals; $24 \%$ of deaths occurred within
3 months of hospital discharge (58\% of these from a general hospital). Only $14 \%$ of those discharged from a general hospital had a recorded psychiatric diagnosis at last visit; an additional $19 \%$ were found to have a previous lifetime psychiatric diagnosis. Median time between last discharge and death was fourfold greater in those without a psychiatric history. Diagnoses also revealed that less than half of those last discharged from general hospital had had a main diagnosis of 'injury or poisoning'.

\section{Conclusions}

Suicide prevention activity, including a better psychiatric evaluation of patients within general hospital settings deserves more attention. Improved information flow between secondary and primary care could be facilitated by exploiting electronic records of previous psychiatric diagnoses.

\section{Declaration of interest}

None.
Suicide is a significant public health concern in the UK and worldwide. Contact with healthcare providers affords opportunities to engage in suicide prevention and considerable interest exists in establishing predictive information on which individuals accessing healthcare services are most at risk. Consequently, studies have explored suicide after discharge from psychiatric hospitals, consistently reporting a peak incidence of suicide within 28 days, ${ }^{1-4}$ and a quarter of deaths occurring within 3 months, of discharge. ${ }^{5}$ Only a few studies considered suicide after discharge from pooled general and psychiatric hospital settings, highlighting that most people had been in contact with healthcare services in the year prior to death ${ }^{6,7}$ and that an estimated $7 \%$ and $13 \%$ had been discharged within 1 month prior to death from general and psychiatric hospitals respectively. ${ }^{8}$

This study uses records of completed suicides in Scotland over a 30-year period to analyse patterns after last discharge by hospital type (general or psychiatric) and according to summary categories (psychiatric diagnosis or in a category of 'injuries or poisonings' coded at last general hospital episode). This UK-based study is one of the largest to be reported, and we aimed to identify whether there were potential alternative opportunities for suicide prevention.

\section{Method}

\section{Study data}

An extraction was made from the National Records of Scotland (NRS) deaths register for the period from 1981 to 2010 of deaths of people aged 15 years or older with underlying cause of death coded as suicide as a result of intentional self-harm (ICD-9 E950-959; ICD-10 X60-84, Y87.0). ${ }^{9,10}$ The lower age cut-off was selected as it follows other UK epidemiological studies of suicide. ${ }^{11-13}$ Although it is conventional research practice to subsume deaths of 'undetermined intent' within an overall category of suicides, this study focused on those deaths that had sufficient proof of suicidal intent ('as a result of intentional selfharm'). The NRS data-set could have been increased in size by $39 \%$ by adding in deaths coded as 'undetermined events', however we had no problems associated with sparse data, a common feature of other suicide studies, and excluding undetermined events also ruled out any possibility of including 'false positive' data.

This study capitalises on Scotland's privileged position as one of a handful of countries that has digitised National Health Service (NHS) hospital records that are linkable by a unique patientidentifiable number (Community Health Index introduced in 1981). ${ }^{14}$ Up to 30 years of hospital-episode data for all deaths by suicide was linked using the 'Scottish Morbidity Records' in-patient and day-case physical health episodes ('SMR01'), and in-patient and day-case mental health episodes ('SMR04'). Out-patient and maternity records were not included in the data-set.

Problems of data quality are a well-known limitation of administrative data sources - errors have the potential to arise, for instance, because of coding or transcription errors in the original production of data, and secondary researchers have little opportunity to identify or address errors. A review of the data revealed some anomalies that were likely to reflect data errors. For instance, a number of the hospital episodes stemming from individuals were erroneous and had to be excluded; some had the wrong linked hospital records, some records were duplicates, whereas others had valid records but contained month of 
admission later than month of death, suggesting random Community Health Index or date transcription errors. Excluded records are reported within the study results.

\section{Data processing and variable operationalisation}

Hospital episodes were coded on main and secondary diagnoses contained in ICD $-9^{9}$ from 1981 to 1996 , with a change to ICD-10 ${ }^{10}$ from 1 April 1996. Each record contained a main diagnosis and up to ten possible secondary diagnoses. Summarising diagnostic information at hospital visits necessitated harmonising ICD codes to obtain consistent coding over time. We achieved this by collapsing ICD codes into broader, clinically homogeneous categories, using a tool called Clinical Classifications Software (CCS) on Windows 7 Enterprise. Clinical Classifications Software is part of a sponsored project by the US Agency for Healthcare Research and Quality and is available to download. ${ }^{15,16}$ We used the 'special archival' single-level diagnosis version, aggregating all ICD-9 and ICD-10 diagnostic codes in our linked data-set into 260 CCS meta-categories.

The linkage between ICD codes and CCS summary codes required ICD codes to be stored in highly standardised formats. Therefore, as is common practice when working with administratively collected data, substantial cleaning of the original data was required to achieve this linkage (for example, of 'main diagnosis' ICD records across all data-sets, $68 \%$ of all ICD codes were re-processed in some manner to allow them to link with the ICD formats used in the CCS tool). Extended algorithms were written using Stata V11 on Windows 7 Enterprise, featuring tasks such as removing spaces and 'wildcard' indicators such as ' $\mathrm{X}$ ', and changing when relevant the number of characters of detail, in order to achieve a standardised ICD format. The Stata software code used to do this (including the macros used to translate in turn from ICD to CCS codes) has been published by the research team so that it is available for other researchers. The code files are available at www.dames.org.uk/docs/papers/appendices/smr_icd/. These files have also been submitted to the UK's 'Administrative Data Liaison Service' (www.adls.ac.uk), which provides an online pool for software code relevant to processing administrative data.

Following this processing exercise, approximately $5 \%$ of the codes across the data-set could still not be successfully matched to relevant ICD standardised codes as used in the CCS tool; for instance $90 \%$ of these codes did not contain the expected alphanumeric values and the remaining $10 \%$ were conventionally formatted ICD codes but linked to no known ICD standard. Both patterns are consistent with data-processing errors at the point of data collection that might be regarded as occurring randomly.

From the available diagnostic information designated by the 260 CCS codes, we used relevant categories for psychiatric diagnosis (CCS codes $65-75$, corresponding to a broad category approximately equating to codes 290-319 from ICD-9 and chapter F, ICD-10). For self-harm we were restricted to using broad CCS proxy categories of 'any injury or poisoning. On SMR01, codes for 'injury or poisoning' generally appear as the main diagnosis code, equating to CCS codes 225-244, with external causes of harm (ICD-9 E-codes; ICD-10 X- and Y-codes) appearing only as additional secondary diagnoses, equating to CCS code 260. These codes include injury or poisoning of accidental origin as well as assault or self-harm. Although not ideal in terms of focusing specifically on self-harming behaviour, we decided to use these codes in establishing any relative differences between demographic groups.

Death record variables used included age, gender, marital status (married/cohabiting or not) and employment status ('not in work' or otherwise). We also obtained a linked Carstairs Index quintile variable, a local area-based measure of deprivation ${ }^{17}$ that was available for the entire time span of the data-set. Values were individually assigned using the postcode recorded on the death certificate. Descriptive data analysis was used in summarising group characteristics and proportions of people who died after different time points post last hospital discharge. We used Stata V11 on Windows 7 Enterprise throughout.

This study was approved by Lothian Research Ethics Committee (No.10/S1103/31), the Privacy Advisory Committee (PAC) of NHS Information Services Division and the Registrar General of NRS. Caldicott Guardian approval was granted as part of the PAC approval.

\section{Results}

\section{Overall cohort characteristics}

The study time span, 1981-2010, covered 16475 deaths by suicide, consisting of 12162 (74\%) males and 4313 (26\%) females. There was a time-lag between deaths recorded and being linked to hospital records; therefore we truncated the data-set at the end of 2009, leaving 16411 individuals for further analysis, of whom 5275 had no linked hospital records and 229 died in hospital having no previous admission records, leaving 10907 people with a last discharge alive (Table 1).

Individuals who died by suicide with hospital records were predominantly male (72\%); had a mean age of 43 ; more frequently living alone (63\%); had a mean Carstairs quintile of 3.3 (slightly more deprived areas than population average); and $20 \%$ were not in work. Gender interacted with other measures: males were significantly younger at death than females, and significantly more women (34\%) were not in work compared with men $(15 \%)$.

Table 1 Characteristics of people who died by suicide in Scotland 1981-2009 ( $n=16411)$ with and without hospital records (in-patient or day-case episodes)

\begin{tabular}{|c|c|c|c|c|c|c|}
\hline & \multicolumn{3}{|c|}{$\begin{array}{l}\text { People with hospital records and alive at last } \\
\text { hospital discharge }\end{array}$} & \multicolumn{3}{|c|}{$\begin{array}{l}\text { People with no hospital records or who had one } \\
\text { hospital record where the outcome was death }\end{array}$} \\
\hline & All & Men & Women & All & Men & Women \\
\hline Deaths, $n(\%)$ & $10907(100)$ & $7842(72)$ & $3065(28)$ & $5504(100)$ & $4270(78)$ & $1234(22)$ \\
\hline Age at death, years: mean (s.d.) & $43.3(16.8)$ & $42.1(16.4)$ & $46.2^{*}(17.2)$ & $43.4(16.8)$ & $41.8(16.1)$ & $48.8^{*}(18.2)$ \\
\hline Married or cohabiting at death, \% & 37.2 & 37.0 & 37.6 & 43.3 & 43.1 & 44.1 \\
\hline Carstairs Index, mean ${ }^{a}$ & 3.27 & 3.27 & 3.27 & 3.16 & 3.14 & 3.23 \\
\hline Not in work, ${ }_{1}^{\mathrm{b}} \%$ & 20.0 & 14.5 & $34.1^{*}$ & 14.3 & 9.0 & $32.6^{*}$ \\
\hline \multicolumn{7}{|c|}{$\begin{array}{l}\text { a. The area-based Carstairs index of deprivation quintiles (1991); } 1 \text {, low deprivation to } 5 \text {, high deprivation. } \\
\text { b. National Records of Scotland categories of 'Students, independent means, no occupation, disabled'. } \\
{ }^{*} P<0.05 \text { significant differences by gender at } 95 \% \text { threshold. }\end{array}$} \\
\hline
\end{tabular}


Comparison of people with and without previous hospital records revealed further differences (Table 1). Men had accessed secondary-care services less often than women $(72 \%$ of deaths with previous hospital records were men, compared with $78 \%$ of deaths without previous records). In addition, people with hospital records had more frequently been living alone than those without such records (63\% v. 57\%) and higher proportions of men with records were not in work compared with those without records $(15 \%$ v. $9 \%)$.

\section{Suicide patterns in relation to last discharge from pooled hospital settings}

The 10907 people with psychiatric or general hospital records prior to death had a total of 66188 records. This figure was arrived at having removed 362 records with admission dates after the death dates; 1016 records for being exact duplicate records of another episode and 2002 records where the outcome was death in hospital. The 66188 records comprised 23\% psychiatric hospital records, however total population data from NHS Information Services Division estimates that 32.8 million hospital records were created nationwide over the same time span, of which only $3 \%$ were psychiatric records.

Substantially more people who died by suicide were last discharged from general rather than psychiatric hospitals - we identified the last hospital discharge record for each of the 10907 , defining categories for those who had last been in general hospital $(n=8263,76 \%)$ or in psychiatric hospital $(n=2270$, 21\%). A further 374 people had 'tied' records, meaning they had both general and psychiatric hospital admissions and transfers between hospitals within the same last continuous period of care (Table 2).

Of 16411 deaths overall, $50 \%$ had a last discharge from general hospital, $16 \%$ had a last discharge that included a psychiatric hospital record and 34\% had no prior hospital records. A total of $16 \%$ of all deaths by suicide had occurred within 3 months of last discharge from any hospital $(n=2575 / 16411)$, a figure which amounts to about a quarter of all deaths among previous hospital patients $(n=2575 / 10907,24 \%)$ in the same period. Of all deaths, 9\% $(n=1487 / 16411)$ had been discharged within 3 months from a general hospital, and a further $7 \%$ $(n=180+908 / 16411)$ had been discharged within 3 months from a psychiatric hospital (including combined settings). Similarly, $32 \%$ of all deaths in Scotland by suicide were of those who had been discharged from a hospital within the last year prior to death $(n=5178 / 16411)$; three-fifths of these $(n=3312)$ happened after general hospital discharge and did not involve a linked psychiatric episode.

Of the whole population who died by suicide, about half of all male and female deaths occurred after last discharge from general hospital (51\% and $49 \%$ respectively). However, around $14 \%$ of male compared with $22 \%$ of female deaths had occurred after last discharge from psychiatric hospital (or a continuous episode of care of both a general and a psychiatric hospital). This disparity may well reflect that higher numbers of women are generally admitted to psychiatric hospital in the first place, but may nevertheless be indicative of how suicide prevention efforts should be sensitive to differences between the experiences of men and women in the hospital system.

\section{Last discharge from general hospitals with a diagnosis of injury or poisoning}

Given the high volume of deaths arising after a last discharge from general hospitals, we set out to provide high-level summaries of whether people with a last discharge alive from a general hospital had been diagnosed with 'injury or poisoning', an aggregate category of assaults, accidents and self-injuries.

Less than half of all individuals ( $n=3179,38 \%$; Table 3 ) who died after last discharge from general hospital had been given a main or any secondary diagnosis of 'injury or poisoning', of whom $22 \%(n=705)$ had completed suicide within 3 months of last discharge, $33 \%(n=1047 / 3179)$ by 6 months and two-thirds ( $n=2154)$ within 1 year of last discharge.

Young men were the most prominent group in this category: men aged 15-45 comprised by far the largest category of people who died after a last discharge with a diagnosis of injury or poisoning' in general hospital (60\% of all deaths, $n=1917)$. By comparison, $19 \%$ of deaths from this group were of men aged over 45 ; whereas $13 \%$ were of women aged $15-45$ and $9 \%$ were women over 45 (Table 3).

Among those deaths that occurred after last discharge from general hospital, we found that $62 \% \quad(n=5125 / 8263)$ had previously received a diagnosis of 'injury or poisoning' in any of their previous hospital records over their lifetime (within the observation period 1981-2009). Indeed, of those with a last discharge following a diagnosis of 'injury or poisoning', $41 \%$ ( $n=1313 / 3179)$ had either a psychiatric disorder recorded at last hospital visit, or had a psychiatric diagnosis included at any time previously in their hospital records.

It seems likely therefore that there is potential to identify an at-risk population in general hospital settings where prevention work or mental health assessment could take place for those not being currently admitted for 'injury or poisoning' but who have had any previous lifetime record for 'injury or poisoning' or psychiatric disorder (or both), and would otherwise appear as 'invisible' to healthcare staff at time of any current admission.

Comparing demographic data for those with a last diagnosis of injury or poisoning (Table 3) with all deaths that had linked hospital records (Table 1), we found that slightly lower

Table 2 The nature of last recorded hospital discharge prior to suicide, Scotland 1981-2009

\begin{tabular}{|c|c|c|c|c|c|c|}
\hline & All suicides, $n(\%)$ & Males, $n(\%)$ & Females, $n(\%)$ & $\begin{array}{l}\text { Suicides within } \\
3 \text { months of } \\
\text { discharge, } n(\%)\end{array}$ & $\begin{array}{l}\text { Suicides within } \\
6 \text { months of } \\
\text { discharge, } n(\%)\end{array}$ & $\begin{array}{l}\text { Suicides within } \\
12 \text { months of } \\
\text { discharge, } n(\%)\end{array}$ \\
\hline Last discharge from psychiatric hospital & $2270^{a}(14)$ & $1458(12)$ & $812(19)$ & $908(35)$ & $1254(33)$ & $1581(31)$ \\
\hline Last discharge from general hospital & $8263^{\mathrm{a}}(50)$ & $6151(51)$ & $2112(49)$ & $1487(58)$ & $2295(61)$ & $3312(64)$ \\
\hline $\begin{array}{l}\text { Last discharge month included psychiatric } \\
\text { and general hospital episodes }\end{array}$ & $374(2)$ & $233(2)$ & $141(3)$ & $180(7)$ & $237(6)$ & $285(6)$ \\
\hline No prior hospital records & $5504(34)$ & $4270(35)$ & $1234(29)$ & - & - & - \\
\hline Subtotals & $16411(100)$ & $12112(100)$ & $4299(100)$ & $2575(100)$ & $3786(100)$ & $5178(101)$ \\
\hline
\end{tabular}




\begin{tabular}{|c|c|c|}
\hline & $\begin{array}{l}\text { Deaths with last discharge from } \\
\text { general hospital with a diagnosis } \\
\text { of 'injury or poisoning', } n \text { (\%) }\end{array}$ & $\begin{array}{l}\text { Deaths with a diagnosis of 'injury or } \\
\text { poisoning' as \% of all deaths with } \\
\text { last discharge from general hospital, \% } \\
\qquad(n=8263)\end{array}$ \\
\hline All & $3179(100)$ & 38 \\
\hline \multicolumn{3}{|l|}{ Gender } \\
\hline Males & $2490(78)$ & 30 \\
\hline Females & $689(22)$ & 8 \\
\hline \multicolumn{3}{|l|}{ Age, years } \\
\hline Males, 15-45 & $1917(60)$ & 23 \\
\hline Males $>45$ & $617(19)$ & 7 \\
\hline Females 15-45 & $421(13)$ & 5 \\
\hline Females $>45$ & $283(9)$ & 3 \\
\hline \multicolumn{3}{|l|}{ Suicides by time of discharge from general hospital } \\
\hline Within 3 months & $705(22)$ & 9 \\
\hline Within 6 months & 1047 (33) & 13 \\
\hline Within 12 months & $2154(68)$ & 26 \\
\hline Any psychiatric disorder at last hospital visit or previous point in lifetime & $1313(41)$ & 16 \\
\hline Married/cohabiting & $972(30)$ & 12 \\
\hline Not in labour market ${ }^{\mathrm{b}}$ & $658(21)$ & 8 \\
\hline In most deprived Carstairs Index quintile (quintile 5) & $913(29)$ & 11 \\
\hline
\end{tabular}

proportions were cohabiting than not (30\% compared with $37 \%$ respectively); but there were no other noticeable differences.

\section{Last discharge from general hospitals and psychiatric morbidity prevalence}

Psychiatric disorders may also be relevant to suicide patterns for those with a last discharge from general hospital. We found that $14 \%$ of all those who completed suicide after last being discharged from general hospital had a psychiatric disorder recorded at the last hospital episode (either as a main or secondary diagnosis). This group had a median time to death of 7 months from last discharge, suggesting that this is an important risk indicator (Table 4).

The category of people who had no psychiatric disorder recorded during their last (general hospital) episode, but did have a psychiatric disorder recorded at some point previously in their lifetime amounted to $19 \%(n=1597 / 8263)$ of those last discharged from general hospital, of whom 30\% $(n=450 / 1487)$ died within 3 months of last discharge, and the overall median time until death was 9 months. This indicates that in many instances where a person is admitted to a general hospital, a prior record of psychiatric disorder may be a relevant indicator of suicide risk.
On the other hand, $67 \%$ of those last discharged from general hospital had no psychiatric diagnosis ever recorded anywhere in their hospital records. In contrast to the first two groups, this group had a much longer median time between discharge and death (33 months).

To reiterate: those last discharged from general hospital with the shortest median time until death (7 months) were those who had a recorded comorbid psychiatric diagnosis at last hospital visit, followed by those who did not have a recorded psychiatric diagnosis at last general hospital visit but did have a diagnosis previously within their lifetime (median time to death of 9 months).

The same proportion of men and women had psychiatric disorders recorded at last general hospital visit (14\%; Table 4). However, substantially more men than women who died after a recorded hospital episode had no psychiatric disorders recorded at any point in their lifetime (69\% v. 60\%; Table 4), whereas fewer men $(17 \%)$ compared with women $(26 \%)$ had no psychiatric disorder recorded at last visit but did have a previous psychiatric disorder along the life course, patterns that would be consistent of a relative underrepresentation of men in accessing hospital psychiatric services. Although we had expected to see an increase in hospital records with age in line with a cumulative diagnostic accrual along the lifespan, we found that the proportion of males

\begin{tabular}{|c|c|c|c|c|c|c|}
\hline \multirow[b]{2}{*}{ Time psychiatric disorder recorded } & \multicolumn{5}{|c|}{$n(\%)$} & \multirow{2}{*}{$\begin{array}{l}\text { Months from last } \\
\text { discharge to death } \\
\text { Median (IQR) }\end{array}$} \\
\hline & All & Males & Females & $\begin{array}{l}\text { Males } \\
\text { 15-45 years }\end{array}$ & $\begin{array}{l}\text { Suicide within } \\
3 \text { months of discharge }\end{array}$ & \\
\hline Recorded at last hospital visit & $1126(14)$ & $833(14)$ & $293(14)$ & $543(14)$ & $365(25)$ & $7(3-24)$ \\
\hline Recorded, but not at last visit & $1597(19)$ & $1053(17)$ & $544(26)$ & $656(17)$ & $450(30)$ & $9(3-24)$ \\
\hline None recorded at any time in lifetime & $5540(67)$ & $4265(69)$ & $1275(60)$ & $2687(69)$ & $672(45)$ & $33(9-81)$ \\
\hline Subtotals & $8263(100)$ & $6151(100)$ & $2112(100)$ & $3886(100)$ & $1487(100)$ & $20(6-60)$ \\
\hline
\end{tabular}


aged 15-45, who were last discharged from general hospital with no psychiatric disorder, was identical $(69 \%)$ to the aggregate category of all males with no psychiatric disorder.

Lastly, Fig. 1 shows the percentage of suicides occurring within different time spans since last discharge from general hospitals (recorded in SMR01) or from psychiatric hospitals (recorded in SMR04). Although fewer suicides involve last discharge from a psychiatric hospital, the temporal pattern suggests that most suicides involving people discharged from such facilities occurred in relatively shorter time periods since the last discharge. This is in contrast to the pattern of suicide following discharge from a general hospital, where relatively more deaths occur at longer intervals since the last recorded discharge. This is not so surprising if admission/readmission patterns to hospital are related to suicide risk and merits further analysis of hospital utilisation patterns prior to suicide.

\section{Discussion}

\section{Main findings}

This study reports on a large linked UK data-set of individuals who died from suicide, spanning 1981-2009. One-third of those who died by suicide had no previous hospital records whatsoever (although there is the possibility of previous hospital admissions in another country), and half of all deaths by suicide were of people who had been last discharged from general hospital.

Deaths by suicide in individuals with hospital records were more than three times more frequently last discharged from a general hospital than a psychiatric hospital. This was higher than anticipated, but is perhaps not that surprising since there are many more admissions to general than psychiatric hospital among the national population. It is revealing however, to compare the time spans between discharge and death in relation to last discharge type; many deaths occurred relatively soon after a previous discharge from either category, suggesting opportunities for intervention may exist in both settings. Examination of diagnoses revealed that less than half of those last discharged from general hospital had received a diagnosis of 'injury or poisoning'; potentially suggestive of subsequent self-harm, with more than half of these being younger men.

Despite having died by suicide, we found only a small minority (14\%) had a recorded comorbid psychiatric diagnosis at last general hospital visit, but that an additional 19\% had received a previous psychiatric diagnosis during their lifetime; the median times to death when there was a psychiatric diagnosis at last discharge was 7 months, and 9 months for those with any previous psychiatric history; this was in marked contrast to 33 months for people who had no (hospital) psychiatric diagnosis history whatsoever.

\section{Limitations of the study}

The general hospital data-set (SMR01) permits identification of individuals transferred to other NHS hospitals and psychiatric facilities but does not allow for capturing hospital episodes of people who present to accident and emergency departments and who subsequently self-discharge or who are not then admitted to hospital. Therefore it is likely that there is an underrepresentation of people in crisis in the data-set who have presented to hospital; the numbers dying by suicide after contact with general hospitals are likely to be higher than this analysis suggests.

This study does not link to primary care or hospital out-patient data. An analysis of all secondary- and primary-care linked records would have provided a more comprehensive approach in summarising healthcare contacts prior to suicide; however, this was beyond the scope of this study. The data used for this study do not contain deaths deemed to be 'events of undetermined intent' and we recognise that in ruling out possible 'false positive' suicide deaths, we have potentially missed many additional genuine records (estimated as an additional 6640 individuals for the time span 1981 to 2010; see NRS official statistics, www. gro-scotland.gov.uk/statistics/theme/vital-events/deaths/suicides/ index.html).

With respect to using the broad category of injury or poisoning', this approach was suboptimal as a proxy for self-harm as the data were conflated with all accidents and assaults. However, this makes the low numbers of people last discharged from a general hospital with a diagnosis of 'injury or poisoning' even more surprising. There remains potential to use more detailed
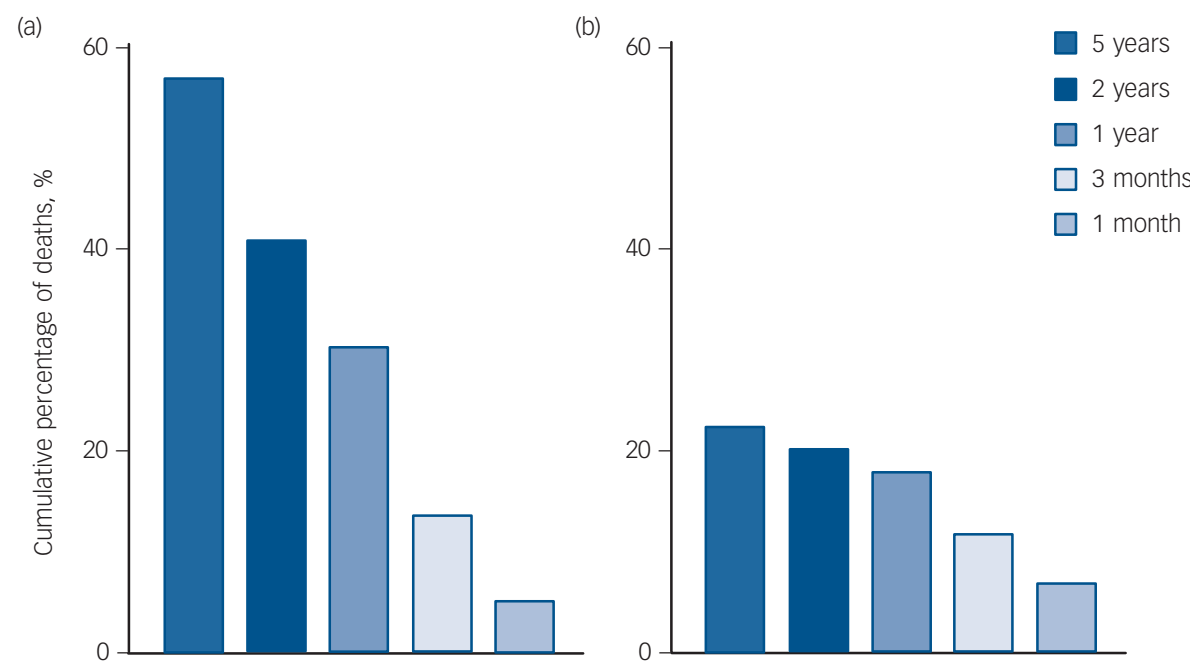

Time since last discharge

Fig. 1 Temporal patterns of suicide after last discharge alive from (a) general hospital or (b) psychiatric hospital, expressed as \% of all suicides $(n=10907)$.

Data are included in (a) if the last hospital record was coded general hospital (SMR01) and included in (b) if the last hospital record was coded psychiatric hospital (SMRO4) or if it was a tied record, i.e. admitted to general hospital (SMR01) and transferred to psychiatric hospital (SMRO4) in continuous episodes of care. 
ICD information (rather than the CCS categories used) in a future analysis, such as to focus on self-harm only.

We did not restrict the data time span so as to permit an accrual of hospital records for those who died early in the 1980s and soon after the data-set capture of hospital records. This means that the prior hospital episodes of some cases are left-censored (if they had prior episodes before 1981). Such imbalances would be quite typical of any practical system that tried to use administrative data to enhance decision-making, for which reason we think it was appropriate to conduct the analysis in terms of deaths since 1981 and their relationship to hospital records since 1981. We also note that our data included months as the most detailed time point for an episode - this raised practical, but not insurmountable, problems in determining the last episode (if more than one episode was held in the same month), although again this is commensurate with the sort of data resources that might be pragmatically available. In addition, there may be significant disclosure risks were data provided identifying exact dates of hospital episodes and deaths.

Finally, for operational reasons the data-set we used was restricted to deaths by suicide. Although a strength of the data is the nationwide coverage (with no inherent selection bias), this was a single group study, and in further work a comparison of this group with other non-suicide cohorts or case-controls has obvious attractions and could yield important information on relative risks.

\section{Comparison with previous studies}

Studies exploring suicide patterns after last discharge from pooled clinical settings are relatively scarce and most do not have control groups. Where studies exist, these tend to focus on patterns after discharge from psychiatric facilities, after episodes of self-harming behaviour, or in estimating the number and frequency of contacts with health services in the time prior to suicide. Analyses suggest that at least $80 \%$ of people who die by suicide had some contact with healthcare services in the year prior to death, ${ }^{6,7,18}$ with more than double the number of contacts being made for those who died by suicide compared with those who did not; ${ }^{7}$ however, it remains unclear whether these contacts with healthcare services had a role in suicide prevention by delaying the event.

Andersen et al pooled hospital and primary care records in Denmark to report that within the last month before death, $66 \%$ had consulted a general practitioner and $7 \%$ and $13 \%$ had been discharged from general and psychiatric hospitals respectively. ${ }^{8}$ We report a different pattern to that of Andersen et al, namely that in the 3 months before death, 9\% and 7\% had been discharged from general and psychiatric hospitals respectively. These differences could in part be explained by the much smaller sample size in the Andersen et al study $(n=472)$ but could also be a feature of the data time span, which was restricted to the early 1990s, a period during which many European countries replaced older psychiatric institutions with community mental health services.

We also found that the percentage of deaths from suicide after last discharge from general hospital continued to rise over time, in a different pattern to that after admission to a psychiatric hospital, which appeared to decrease in a more exponential pattern with time since discharge, a pattern reported elsewhere. ${ }^{19}$ Considering only those deaths by suicide following last discharge from psychiatric hospitals, a peak incidence of suicide occurred within the 3 months following discharge, in agreement with other studies that report peak incidences within a month after discharge. ${ }^{1-4,20}$ However, a report from Sweden suggests that the majority of deaths with psychiatric diagnosis had been discharged from psychiatric treatment for more than 1 month before suicide ${ }^{21}$ and that 'doctor shopping' behaviour can vary markedly by age and gender with, for example, women's contact with psychiatrists increasing during the year prior to suicide then dropping significantly shortly before suicide. ${ }^{22}$

\section{Future research}

An analysis of data incorporating other healthcare records (for example primary care, emergency department, ambulance service) would offer a more comprehensive understanding of patterns of service utilisation. Although this study summarises only secondary-care records, there are initiatives in pooling data sources, creating richer sources of information that may afford opportunities to glean further insights. ${ }^{23}$ Data-sets such as the one used in this analysis are rich sources of information and yield further opportunities in, for example, summarising morbidity burden, prevalent diagnoses along the life course, temporal patterns in suicide or in making more use of individual-level data such as ICD codes for self-harm or occupation held in the death record.

Further exploration of reasons why relatively more men than women who die by suicide have no psychiatric records is warranted, and also whether this apparent underrepresentation of men accessing hospital psychiatric services is related to gender differences in help-seeking behaviour within primary care.

\section{Implications for practice}

This study highlights that engaging in suicide prevention within the general hospital setting deserves much more attention. Targeted follow-up of people admitted to general hospital with self-harm diagnoses (in particular younger men), and screening for psychological problems in those where there has been either a history of self-harming or of any psychiatric condition along the life course could be beneficial. This would require making better use of historical and electronic health records and ensuring all hospital staff could engage in some first-line mental health screening in these 'at risk' patients.

Although we found that the majority of people discharged from general hospital had no recorded psychiatric morbidity in hospital records, this does not preclude the possibility of psychiatric problems being managed exclusively in primary care. There is potential for better information flow from primary to secondary care (such as relevant episodes of depression or anxiety) to yield useful additional clinical insights, which could be particularly relevant for unscheduled admissions. Many of the people in the study had no hospital records at all, which also highlights the need for adequate community and primary care mental health resources.

In summary, we report that higher percentages of people died by suicide after 3, 6 and 12 months after last discharge from general than from psychiatric hospitals. Less than half of these had a main diagnosis of 'injury of poisoning', a category in which young men were most affected. The median time to death was about fourfold longer for those without any current or previous psychiatric history. More could be done in engaging with suicide intervention, psychiatric evaluation and follow-up of people who are admitted to general hospitals with self-harm history or psychiatric comorbidity, past or present.

\section{Funding}

We thank the Economic and Social Research Council (Grant number RES-149-25-1066) and the Chief Scientist Office of the Scottish Government for part-funding this study. 


\section{Acknowledgements}

We gratefully acknowledge helpful advice given by Frank Dixon, National Records of Scotland.

Nadine Dougall, MSC, NMAHP Research Unit, University of Stirling, Stirling; Pau Lambert, PhD, School of Applied Social Science, University of Stirling, Stirling; Margaret Maxwell, PhD, NMAHP Research Unit, University of Stirling, Stirling Alison Dawson, PhD, School of Applied Social Science, University of Stirling, Stirling Richard Sinnott, PhD, University of Melbourne, Melbourne; Susan Mccafferty, MSc, National escience Centre, University of Glasgow, Glasgow; Carole Morris, BSC, MSC, National eScience Centre, University of Glasgow, Glasgow; Carole Morris, BSC,
David Clark BSc, Anthea Springbett, PhD, Information Services Division, NHS David Clark BSC, Anthea Springbett, P
National Services Scotland, Edinburgh, UK

Correspondence: Nadine J. Dougall, NMAHP Research Unit, School of Nursing Midwifery \& Health, University of Stirling, Unit 13 Scion House, Stirling University Innovation Park, FK9 4NF, UK. Email: nadine.dougall@stir.ac.uk

First received 11 Oct 2012, final revision 27 Jun 2013, accepted 5 Sep 2013

\section{References}

1 Goldacre M, Seagroatt V, Hawton K. Suicide after-discharge from psychiatric inpatient care. Lancet 1993; 342: 283-6.

2 Geddes JR, Juszczak E, OBrien F, Kendrick S. Suicide in the 12 months after discharge from psychiatric inpatient care, Scotland 1968-92. J Epidemiol Community Health 1997; 51: 430-4.

3 Qin P. Suicide risk in relation to level of urbanicity - a population-based linkage study. Int J Epidemiol 2005; 34: 846-52.

4 Hunt I, Kapur N, Webb R, Robinson J, Burns J, Shaw J, et al. Suicide in recently discharged psychiatric patients: a case-control study. Psychol Med 2009; 39: 443-9.

5 Appleby L, Shaw J, Amos T, McDonnell R, Harris C, McCann K, et al. Suicide within 12 months of contact with mental health services: national clinical survey. BMJ 1999; 318: 1235-9.

6 Chang $\mathrm{CM}$, Liao SC, Chiang $\mathrm{HC}$, Chen YY, Tseng KC, Chau YL, et al. Gender differences in healthcare service utilisation 1 year before suicide: national record linkage study. Br J Psychiatry 2009; 195: 459-60.

7 Morrison KB, Laing L. Adults' use of health services in the year before death by suicide in Alberta. Health Rep 2011; 22: 15-22.

8 Andersen UA, Andersen M, Rosholm J, Gram L. Contacts to the health care system prior to suicide: a comprehensive analysis using registers for general and psychiatric hospital admissions, contacts to general practitioners and practising specialists and drug prescriptions. Acta Psychiatr Scand 2000; 102: $126-34$.
9 World Health Organization. International Statistical Classification of Diseases and Related Health Problems (ICD-9). WHO, 1978.

10 World Health Organization. The ICD-10 Classification of Mental and Behavioural Disorders: Clinical Descriptions and Diagnostic Guidelines. WHO, 1992.

11 Platt S, Boyle P, Crombie I, Feng Z, Exeter D. The Epidemiology of Suicide in Scotland 1989-2004: An Examination of Temporal Trends and Risk Factors at National and Local Levels. The Scottish Government, 2007 (http:// www.scotland.gov.uk/Publications/2007/03/01145422/0).

12 Stark C, Hopkins P, Gibbs D, Rapson T, Belbin A, Hay A. Trends in suicide in Scotland 1981-1999: age, method and geography. BMC Public Health 2004; 4: 49

13 Middleton N, Whitley E, Frankel S, Dorling D, Sterne J, Gunnell D. Suicide risk in small areas in England and Wales, 1991-1993. Soc Psychiatry Psychiatr Epidemiol 2004; 39: 45-52.

14 Kendrick S, Clarke J. The Scottish Record Linkage System. Health Bull 1993; 51: 72-9.

15 Elixhauser A, Steiner C, Palmer L. Clinical Classifications Software (CCS), 2012. US Agency for Healthcare Research and Quality, 2012 (http:// www.hcup-us.ahrq.gov/toolssoftware/ccs/ccs.jsp).

16 US Agency for Healthcare Research and Quality. Clinical Classifications Software (CCS) for ICD-10. US Agency for Healthcare Research and Quality, 2009 (http://www.hcup-us.ahrq.gov/toolssoftware/icd_10/ccs_icd_10.jsp).

17 Carstairs V, Morris R. Deprivation: explaining differences in mortality between Scotland and England and Wales. BMJ 1989; 299: 886-9.

18 Cho J, Kang D, Moon K, Holman J, Jablensky A, Fuller S, et al. Age and gender differences in medical care utilization prior to suicide. J Affect Disord 2013; 146: $181-8$.

19 Lawrence D, D'Arcy C, Holman J, Jablensky AV, Fuller SA, Stoney AJ. Increasing rates of suicide in Western Australian psychiatric patients: a record linkage study. Acta Psychiatr Scand 2001; 104: 443-51.

20 Deisenhammer E, Huber M, Kemmler G, Weiss E, Hinterhuber H. Psychiatric hospitalisations during the last 12 months before suicide. Gen Hosp Psychiatry 2007; 29: 63-5.

21 Reutfors J, Brandt L, Ekbom A, Isacsson G, Sparén P, Osby U. Suicide and hospitalization for mental disorders in Sweden: a population-based casecontrol study. J Psychiatric Res 2010; 44: 741-7.

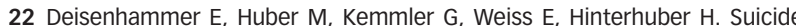
victims' contacts with physicians during the year before death. Eur Arch Psychiatry Clin Neurosci 2007; 257: 480-5.

23 NHS Information Services Division. The Scottish Suicide Information Database (ScotSID) Report 2011. NHS Information Services Division, 2011 (http:// www. isdscotland.org/Health-Topics/Public-Health/Publications/2011-12-20/2 011-12-20-Suicide-Summary.pdf). 